\title{
Cancer antigen-125 and outcomes in acute heart failure: a systematic review and meta-analysis
}

\author{
Ka Hou Christien Li, ${ }^{1,2,3}$ Mengqi Gong, ${ }^{4}$ Guangping Li, ${ }^{4}$ Adrian Baranchuk, ${ }_{1}^{5}$ Tong Liu, ${ }^{4}$ \\ Martin C S Wong, ${ }_{1}^{6}$ Aaron Jesuthasan, ${ }^{3}$ Rachel W C Lai, ${ }^{1,2}$ Jenny Chi Ling Lai, ${ }^{1,2}$ \\ Alex Pui Wai Lee, 1,2 Antoni Bayés-Genis, ${ }^{7,8}$ Rafael de la Espriella, ${ }^{9,10,11}$ \\ Juan Sanchis, ${ }^{9,10,11}$ William K K Wu, ${ }^{2,12,13}$ Gary Tse, ${ }^{1,2,13}$ Julio Nuñez, ${ }^{9,10}$ On behalf of \\ International Health Informatics Study (IHIS) Network
}

\begin{abstract}
- Additional material is published online only. To view please visit the journal online (http://dx.doi.org/10.1136/ heartasia-2018-011044).
\end{abstract}

For numbered affiliations see end of article.

\section{Correspondence to}

Dr Gary Tse, Faculty of Medicine, Chinese University of Hong Kong, Hong Kong, China; tseg@ cuhk.edu.hk

Professor William K K Wu, Department of Anaesthesia and Intensive Care, State Key Laboratory of Digestive Disease, The Chinese University of Hong Kong, Hong Kong, China; wukakei@cuhk.edu.hk

Dr Julio Nuñez, Cardiology Department, Hospital Clínico Universitario de Valencia INCLIVA, Valencia, Spain; yulnunez@gmail.com

$\mathrm{KHCL}$ and $\mathrm{MG}$ are joint first authors.

For Presented at statement see end of article.

Received 29 March 2018 Revised 30 July 2018

Accepted 2 August 2018

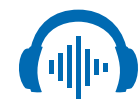

Listen to Podcast heartasia.bmj.com

Check for updates

(c) Author(s) (or their employer(s)) 2018. No commercial re-use. See rights and permissions. Published by BMJ.

To cite: Li KHC, Gong M,

$\mathrm{Li} \mathrm{G}$, et al. Heart Asia

2018:10:e011044

doi:10.1136/

heartasia-2018-011044

\section{ABSTRACT}

Background Carbohydrate antigen-125 (CA125) is an ovarian cancer marker, but recent work has examined its role in risk stratification in heart failure. A recent meta-analysis examined its prognostic value in heart failure generally. However, there has been no systematic evaluation of its role specifically in acute heart failure (AHF).

Methods PubMed and EMBASE databases were searched until 11 May 2018 for studies that evaluated the prognostic value of CA125 in AHF.

Results A total of 129 and 179 entries were retrieved from PubMed and EMBASE. Sixteen studies (15 cohort studies, 1 randomised trial) including 8401 subjects with AHF (mean age 71 years old, $52 \%$ male, mean followup 13 months, range of patients $525.1 \pm 598.2$ ) were included. High CA125 levels were associated with a $68 \%$ increase in all-cause mortality (8 studies, HRs: 1.68 , $95 \% \mathrm{Cl} 1.36$ to $2.07 ; \mathrm{p}<0.0001 ; 1^{2}: 74 \%$ ) and $77 \%$ increase in heart failure-related readmissions ( 5 studies, HRs: $1.77,95 \% \mathrm{Cl} 1.22$ to $2.59 ; p<0.01 ; I^{2}: 73 \%$ ). CA125 levels were higher in patients with fluid overload symptoms and signs compared with those without them, with a mean difference of $54.8 \mathrm{U} / \mathrm{mL}$ (5 studies, SE: 13.2 $\mathrm{U} / \mathrm{mL} ; \mathrm{p}<0.0001 ; \mathrm{I}^{2}:$ 78\%)

Conclusion Our meta-analysis found that high CA125 levels are associated with AHF symptoms, heart failurerelated hospital readmissions and all-cause mortality. Therefore, CA125 emerges as a useful risk stratification tool for identifying high-risk patients with more severe fluid overload, as well as for monitoring following an AHF episode.

\section{INTRODUCTION}

Heart failure (HF) is a syndrome characterised by ventricular dysfunction and dilatation and a compensatory increase in systemic vascular resistance due to neurohumoural activation. ${ }^{1}$ Moreover, a proinflammatory environment with altered metabolic substrate utilisation is present. ${ }^{2} \mathrm{HF}$ is a major public health problem globally, with a prevalence of 5.8 million in the USA and more than 23 million worldwide. ${ }^{3}$ Therefore, it places a burden on healthcare systems, accounting for $1 \%-2 \%$ of the healthcare expenditure. ${ }^{4}$ Of the total costs, approximately $75 \%$ is due to hospital admissions. Rehospitalisation following a hospitalisation for acute heart failure (AHF) is around $20 \%$ at 1 month, rising to $50 \%$ at 6 months. ${ }^{5}$ A history of

\section{Key messages}

What is already known about this subject?

- Carbohydrate antigen-125 (CA125) is an ovarian cancer marker, and recent research work has focused on its role in risk stratification in heart failure.

- A recent meta-analysis examined its prognostic value in heart failure generally, but not specifically in acute heart failure (AHF).

What does this study add?

- Our meta-analysis found that high CA125 levels are associated with heart failure symptoms, heart failure-related hospital readmissions and all-cause mortality in AHF.

How might this impact on clinical practice?

- CA125 emerges as a useful tool for risk stratification to identify high-risk patients with more severe fluid overload, as well as for monitoring following an episode of AHF.

- A patient-level data meta-analysis would provide additional insight into the value of CA125-guided management for patients with AHF.

hospitalisation is itself an independent predictor of long-term mortality. Therefore, measures to reduce hospitalisation are likely beneficial in this patient population and will significantly reduce the costs associated with AHF admissions. ${ }^{6}$ A stepwise approach is needed to achieve this goal. In this case, an understanding of AHF-related prognostic markers is required before it can be used to identify high-risk patients for appropriate intervention to improve prognostic outcome.

The pathophysiology of AHF is complex and multifactorial. Fluid accumulation and redistribution are found in most patients. ${ }^{7}$ Fluid overload is assessed clinically through symptoms such as dyspnoea and increasing lower oedema, and signs such as raised jugular venous pressure, but their sensitivity and specificity are limited. ${ }^{8}$ Moreover, prognostic factors for AHF are different from those for chronic HF. ${ }^{9}$ Consequently, the search for more accurate markers of congestion to guide treatment and inform prognosis remains an unmet clinical need. For example, plasma level of carbohydrate antigen 125 (CA125), a high-molecular-weight, 
soluble glycoprotein released from coelomic or mullerian epithelium, is long-recognised as a tumour marker of ovarian cancer, ${ }^{10}$ but its clinical role in cardiovascular diseases has only been investigated in recent years. ${ }^{11}$ Mechanical stress, increased venous pressures and inflammatory stimulus can stimulate mesoendothelial cells to release CA125.11-13

Prior to CA125, many other prognostic markers such as natriuretic peptides, high-sensitivity troponin, proadrenomedullin, soluble ST2 and galectin-3 have been assessed. Despite the effectiveness of some of the prognostic markers, the difficulty remains in obtaining determinable predictors in a short time frame for patients with AHF. ${ }^{14}$ Furthermore, a meta-analysis has been published on the prognostic value of CA125 in HF, ${ }^{15}$ but only 4 of the 23 included studies concerned patients with AHF. Given the differing pathophysiology of acute versus chronic HF along with the burden of HF shifting from chronic heart failure (CHF) to AHF, we conducted a systematic review and meta-analysis of the prognostic value of CA125 in predicting hospital readmission and mortality outcomes specifically in AHF. Not only does this complement the prior meta-analysis of HF in general, it also serves to evaluate CA125 as a potential prognostic marker for AHF-related all-cause mortality, readmission and symptoms.

\section{METHODS}

\section{Search strategy, and inclusion and exclusion criteria}

The meta-analysis was performed according to the Preferred Reporting Items for Systematic Reviews and Meta-Analyses statement. ${ }^{16}$ PubMed and EMBASE were searched for studies that investigated the relationship between CA125 levels and AHF using the following terms: $[(((()(($ left ventricular dysfunction) OR cardiac failure) OR cardiac insufficiency) OR AHF) OR CCF) OR heart failure) OR decompensated) OR decompensation) AND (((cancer antigen-125) OR Carbohydrate antigen125) OR CA125)]. The search period was from the beginning of the databases through to 11 May 2018 without language restrictions. The following inclusion criteria were applied: (1) the design was a case-control, prospective or retrospective cohort study in humans, and (2) CA125 values were provided and related to clinical endpoints in AHF.

The quality assessment of these studies included in our meta-analysis was performed using the Newcastle-Ottawa Quality Assessment Scale (NOS). The point score system evaluated the categories of study participant selection, comparability of the results and quality of the outcomes. The following characteristics were assessed: (1) representativeness of the exposed cohort; (2) selection of the non-exposed cohort; (3) ascertainment of exposure; (4) demonstration that outcome of interest was not present at the start of the study; (5) comparability of cohorts on the basis of the design or analysis; (6) assessment of outcomes; (7) follow-up period sufficiently long for outcomes to occur; and (8) adequacy of follow-up of cohorts. Each category was assigned a 0 or 1 score in accordance with the assessment form. The total score varied from 0 to 9 stars, which indicated that studies were graded as poor quality if they received 0 or 1 star in the selection domain OR 0 star in the comparability domain OR 0 or 1 star in the outcome/exposure domain; fair if they received 2 stars in the selection domain AND 1 or 2 stars in the comparability domain AND 2 or 3 stars in the outcome/ exposure domain; and good if they received 3 or 4 stars in the selection domain AND 1 or 2 stars in the comparability domain AND 2 or 3 stars in the outcome/exposure domain. The details of the NOS quality assessment are shown in online supplementary table 1. Quality assessment of randomised controlled trials was performed using the Cochrane Risk Assessment Tool (online supplementary figures 1 and 2).

\section{Data extraction and statistical analysis}

Data from the different studies were entered in prespecified spreadsheet in Microsoft Excel. All publications identified were assessed for compliance with the inclusion criteria. In this meta-analysis, the extracted data elements consisted of (1) publication details: surname of first author and publication year; (2) study design; (3) follow-up duration; (4) quality score; and (5) characteristics of the population, including sample size, gender, age and cut-off point for CA125 levels. Two reviewers (GT and $M G$ ) independently reviewed each included study, and disagreements were resolved by adjudication with input from a third reviewer (TL).

The mean differences in CA125 levels between non-survivors and survivors, and between those with fluid overload symptoms compared with those without these symptoms, were determined and meta-analysed. For the relationship between CA125 levels and mortality or readmission endpoints, multivariate adjusted HRs with 95\% CI were extracted and analysed for each study. When values from multivariate analysis were not available, those from univariate analysis were used. ORs were converted to HRs using the following formula: $\mathrm{HR}=\mathrm{OR} /(1-$ baseline risk + (baseline risk $\times(\mathrm{OR})$ ).

Heterogeneity across studies was determined using Cochran's $\mathrm{Q}$ value, which is the weighted sum of squared differences between individual study effects and the pooled effect across studies, and the $\mathrm{I}^{2}$ statistic from the standard $\chi^{2}$ test, which describes the percentage of the variability in the effect estimates resulting from heterogeneity. $\mathrm{I}^{2}>50 \%$ was considered to reflect significant statistical heterogeneity. The random-effects model using the inverse variance heterogeneity method was used with $\mathrm{I}^{2}>50 \%$. To locate the origin of the heterogeneity, sensitivity analysis excluding one study at a time and subgroup analyses based on different disease conditions and different endpoints were performed. Funnel plots showing SEs or precision against the logarithms of the OR were constructed. Begg and Mazumdar rank correlation test and Egger's test were used to assess for possible publication bias.

\section{RESULTS}

A flow diagram detailing the above search terms with inclusion and exclusion criteria is depicted in figure 1. A total of 129 and 179 entries were retrieved from PubMed and EMBASE. Of these, 16 studies were included in the final meta-analysis. ${ }^{17-32}$ The baseline characteristics of the included studies are detailed in table 1. Fourteen studies were prospective cohort studies, one was a retrospective cohort study and one was a randomised controlled trial. The mean follow-up duration was 13 months. A total of 8401 subjects, with a mean age of 71 years old, $52 \%$ male, were included in this analysis. Forty-eight per cent (data available from 10 studies) showed left ventricular systolic dysfunction. The mean cut-off point for CA125 was $47.7 \pm 16.7$ $\mathrm{U} / \mathrm{mL}$.

\section{CA125 and risk of mortality and HF-related hospital readmissions}

Six studies assessed the relationship between CA125 levels and risk of mortality in patients with AHF, all of whom reported higher risk of mortality with increased CA125 levels (table 1). Our meta-analysis shows that high CA125 levels were associated with 68\% increase in the risk of mortality (HR: 1.44, 95\% 


\section{9 and 179 publications were retrieved from PubMed and Embase}

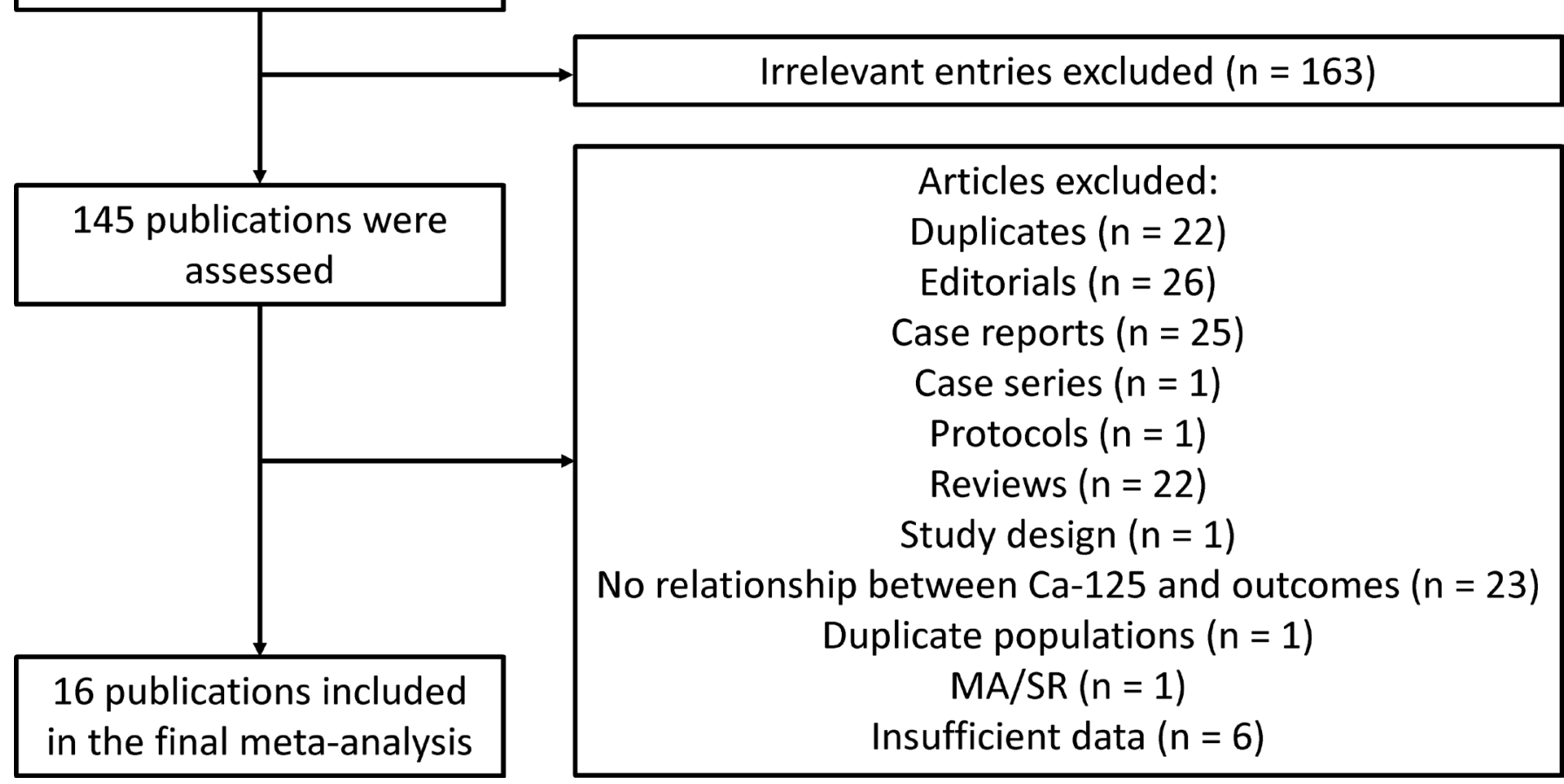

Figure 1 Flow chart of the database search and study selection process for studies evaluating the relationship between carbohydrate antigen-125 (CA125) levels and clinical outcomes in acute heart failure. MA/SR, meta-analysis/systematic review.

\begin{tabular}{|c|c|c|c|c|c|c|}
\hline Studies & Population size (n) & Age \pm SD & Male, n (\%) & Follow-up (months) & HR & $\mathrm{Cl}$ \\
\hline \multicolumn{7}{|c|}{ Outcome all-cause mortality } \\
\hline $\begin{array}{l}\text { Nunez et al }(2015)^{23} \\
\text { (CA125 Gal3) }\end{array}$ & 264 & $72.7 \pm 11.3$ & 131 & 24 & 2.160 & 1.286 to 3.628 \\
\hline Davutoglu et al $(2010)^{17}$ & 100 & $65 \pm 10$ & 59 & 6 & 1.222 & 1.038 to 1.438 \\
\hline Mansour et al $(2010)^{21}$ & 172 & $55.8 \pm 14.5$ & 105 & 40 & 2.840 & $\begin{array}{l}1.651 \text { to } \\
4.884\end{array}$ \\
\hline Jang $(2017)^{25}$ & 457 & $64.4 \pm 16.1$ & 236 & 35.9 & 1.241 & 1.051 to 1.465 \\
\hline $\begin{array}{l}\text { Nunez et al }(2015)^{23} \\
\text { (CA125 BNP) }\end{array}$ & 946 & - & - & 31.68 & 1.340 & 1.148 to 1.564 \\
\hline \multicolumn{7}{|c|}{ Outcome: AHF-associated readmission } \\
\hline \multicolumn{7}{|c|}{ Mean difference in CA125: with pleural effusion versus without pleural effusion } \\
\hline & & & & & $\begin{array}{l}\text { Standard mean } \\
\text { difference }\end{array}$ & SE \\
\hline $\begin{array}{l}\text { Durak-Nalbantic et al } \\
(2013)^{18}\end{array}$ & 50 & $73 \pm 14.6$ & 25 & - & 157.800 & 45.097 \\
\hline Davutoglu et al (2010) $)^{17}$ & 100 & $65 \pm 10$ & 59 & 6 & 36.100 & 7.806 \\
\hline Kouris et al $(2006)^{20}$ & 95 & $70 \pm 10$ & 95 & 15 & 40.000 & 7.469 \\
\hline Ratkovic et al (2016) ${ }^{27}$ & 86 & - & - & - & 97.600 & 17.465 \\
\hline Santas et al $(2015)^{25}$ & 1827 & $72.8 \pm 11.4$ & 895 & 12 & 20.400 & 20.315 \\
\hline
\end{tabular}

AHF, acute heart failure; BNP, brain-type natriuretic peptide; CA125, carbohydrate antigen-125. 
High CA125 levels and mortality in acute heart failure

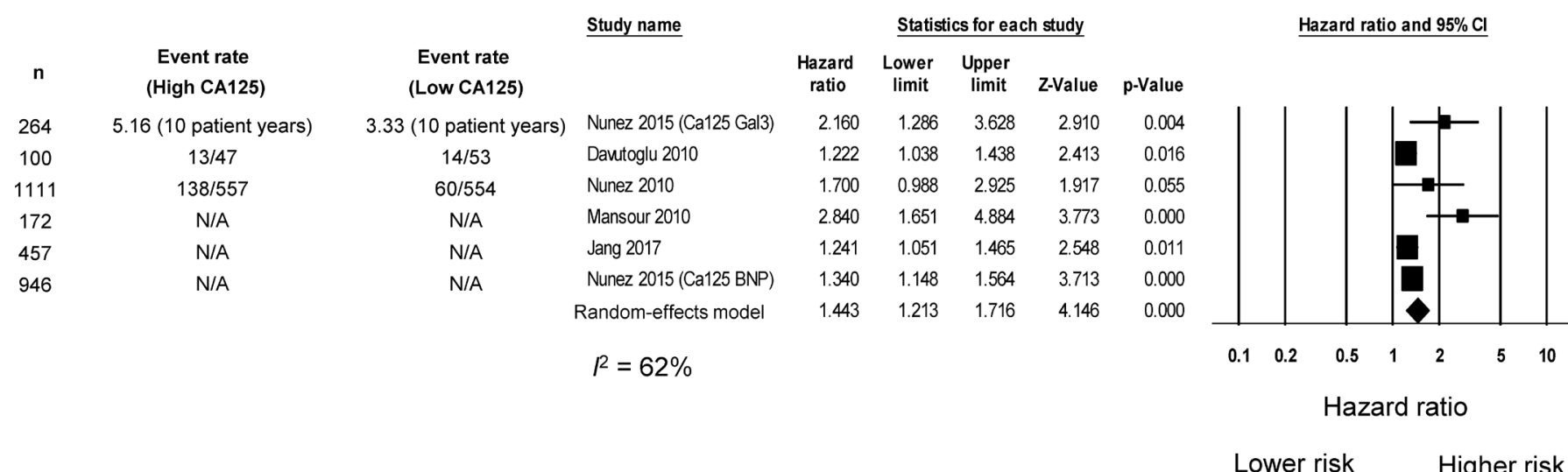

Figure 2 HR for high CA125 levels and mortality in acute heart failure. BNP, brain-typenatriuretic peptide; CA125, carbohydrate antigen-125; N/A, not available.

CI 1.21 to $1.72 ; \mathrm{p}<0.0001$; figure 2 ). $\mathrm{I}^{2}$ took a value of $62 \%$, indicating the presence of moderate heterogeneity. A funnel plot of SE against the logarithm of HR is shown in online supplementary figure 3. Begg and Mazumdar rank correlation without continuity correction suggested the presence of no significant publication bias (Kendall's tau value $0.47, \mathrm{p}=0.19$ ). Egger's test demonstrated significant asymmetry (intercept 2.8 , t-value 3.9 ; $\mathrm{p}<0.05)$. Sensitivity analysis excluding one study at a time did not significantly affect the pooled estimate (online supplementary figure 4).

Three studies assessed the relationship between CA125 levels and risk of HF-related hospital readmissions in patients with AHF, all of whom reported higher risk with increased CA125 levels. Our meta-analysis shows that high CA125 levels were associated with $51 \%$ increase in the risk of HF-related readmissions (HR: $1.51,95 \%$ CI 1.11 to 2.04; $\mathrm{p}<0.01$; figure 3). $\mathrm{I}^{2}$ took a value of $59 \%$, indicating the presence of moderate heterogeneity. A funnel plot of SE against the logarithm of HR is shown in online supplementary figure 5. Begg and Mazumdar rank correlation without continuity correction suggested the presence of no significant publication bias (Kendall's tau value $1, \mathrm{p}=0.12$ ). Egger's test demonstrated no significant asymmetry (intercept 2.2 , $\mathrm{t}$-value $4.1 ; \mathrm{p}=0.15$ ). Sensitivity analysis excluding one study at a time did not significantly affect the pooled estimate (online supplementary figure 6).

Two studies reported on all-cause readmissions, but their study designs were different. One was a randomised controlled trial comparing CA125-guided HF treatment compared with usual care, whereas the other study compared hospitalisations between high and low CA125 cohorts.

\section{CA125 as a marker of congestion severity}

Five studies compared CA125 levels between patients with and without effusion in the context of AHF. Of these, four studies reported significant elevations in this marker with the presence of effusion, whereas the remaining study reported no difference between the two groups (figure 4). The pooled mean difference was $54.8 \mathrm{U} / \mathrm{mL}$ (SE: $13.2 \mathrm{U} / \mathrm{mL}, \mathrm{p}<0.0001) . \mathrm{I}^{2}$ took a value of $78 \%$, indicating the presence of significant heterogeneity. A funnel plot of SE against the logarithm of HR is shown in online supplementary figure 7. Begg and Mazumdar rank correlation suggested no significant publication bias (Kendall's tau value 0.2, $\mathrm{p}>0.05$ ). Egger's test demonstrated no significant asymmetry (intercept 2.5 , t-value $1.5 ; \mathrm{p}>0.05$ ). Once again, sensitivity analysis excluding one study at a time did not significantly affect the pooled estimate (online supplementary figure 8).

\section{DISCUSSION}

The main findings of this meta-analysis are that (1) high CA125 levels are predictive of increased hospital readmissions and mortality in patients with AHF, and (2) CA125 levels are higher in those with pleural effusion. These findings complement and extend those of a recent meta-analysis that

\section{High CA125 levels and HF readmission in acute heart failure}

$\begin{array}{cccl}\text { Event rate } & \text { Event rate } & \text { Study name } \\ 264 & \begin{array}{c}\text { (High CA125) } \\ \text { (Low CA125) }\end{array} & \\ 1111 & 1.4 \text { (per patient years) } & \text { 1.49 (per patient years) } & \text { Nunez 2015 (Ca125 Gal3) } \\ 95 & 208 / 557 & 200 / 554 & \text { Nunez 2010 } \\ & \text { N/A } & \text { N/A } & \text { Kouris 2006 } \\ & & & \text { Random-effects model } \\ & & & \\ & & & \end{array}$

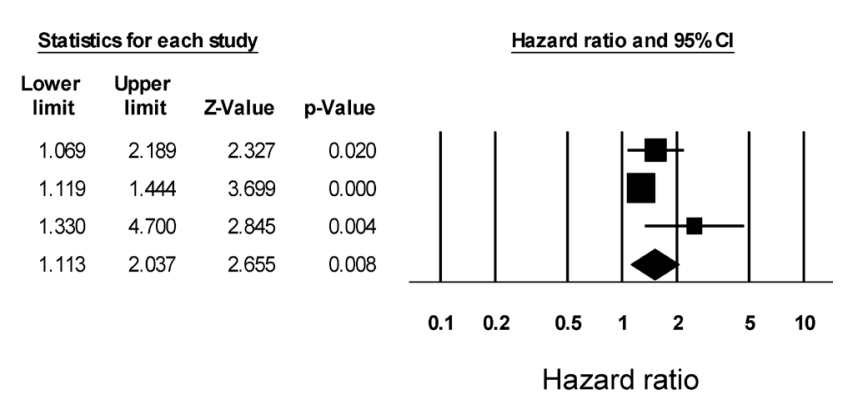

Lower risk

Figure 3 HR for high CA125 levels and hospital readmissions in acute heart failure. CA125, carbohydrate antigen-125; HF, heart failure; N/A, not available. 


\section{Mean difference in Ca-125 levels between patients with and without pleural effusion}

Study name

$\begin{array}{lrr} & \begin{array}{r}\text { Difference } \\ \text { in means }\end{array} & \begin{array}{c}\text { Standard } \\ \text { error }\end{array} \\ \text { Durak-Nalbantic 2013 } & 157.800 & 45.097 \\ \text { Davutoglu 2010 } & 36.100 & 7.806 \\ \text { Kouris 2006 } & 40.000 & 7.469 \\ \text { Ratkovic 2016 } & 97.600 & 17.465 \\ \text { Santas 2016 } & 20.400 & 20.315 \\ \text { Random-effects model } & 54.787 & 13.170\end{array}$

$R^{2}=78 \%$
Statistics for each study

$\begin{array}{rcr}\text { Variance } & \begin{array}{c}\text { Lower } \\ \text { limit }\end{array} & \begin{array}{c}\text { Upper } \\ \text { limit }\end{array} \\ 2033.735 & 69.412 & 246.188 \\ 60.939 & 20.800 & 51.400 \\ 55.782 & 25.362 & 54.638 \\ 305.014 & 63.370 & 131.830 \\ 412.715 & -19.417 & 60.217 \\ 173.439 & 28.975 & 80.599\end{array}$

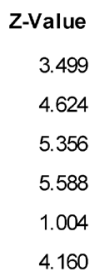

Difference in means and $95 \% \mathrm{Cl}$

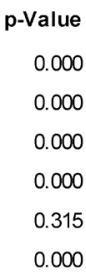

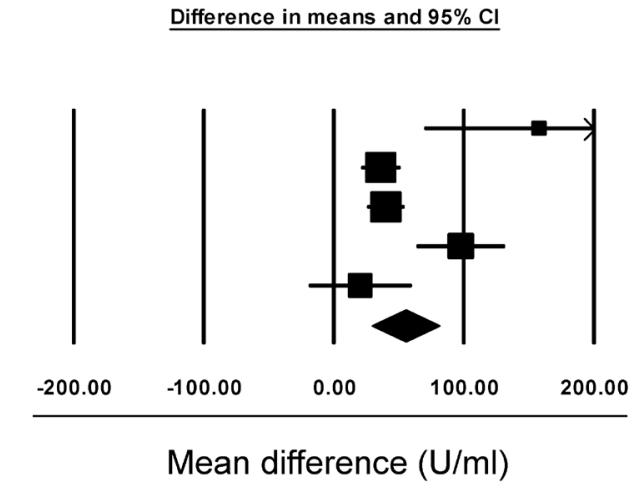

Lower in patients with effusion

\section{Higher in patients with effusion}

Figure 4 Mean difference of carbohydrate antigen-125 (CA125) levels between more severe fluid overload symptoms and those with less symptoms in acute heart failure.

reported the significant prognostic value of CA125 in $\mathrm{HF}^{15}$ and in predicting atrial fibrillation. ${ }^{33}$ The implications are that CA125 may be used to guide clinical management, for example by prescribing higher doses of diuretics for those with high CA125, in whom fluid overload is more severe. It can also be applied synergistically with brain-type natriuretic peptide (BNP), which has shown an association between achieving natriuretic peptide predischarge thresholds and reduced AHF mortality and readmission. ${ }^{34}$

Fluid overload is the hallmark of patients with $\mathrm{AHF}^{7}{ }^{8}$ However, it is well known that congestion is largely heterogeneous, ranging from patients with predominant intravascular redistribution to those with anasarca. ${ }^{7824}$ The rationale for searching new markers of congestion stems from the fact that the traditional tools (symptoms, signs, X-ray and natriuretic peptides) have shown a limited diagnostic accuracy for quantifying the degree of fluid overload in patients with AHF. ${ }^{8}$ Our findings suggest that CA125 levels may provide a more objective assessment of greater fluid overload associated with pleural effusion in patients with AHF, in turn helping to optimise diuretic therapy. ${ }^{3536}$

In the previous meta-analysis, Zhuang et al $^{15}$ assessed CA125 for HF diagnosis, stratification and outcome evaluation of 23 studies, with results in keeping with our analysis. However, the majority of included studies examined chronic HF, with only 4 out of 23 papers relating to AHF, although patients with AHF constituted approximately half of the cohort. Furthermore, the burden of HF has shifted over the recent years from $\mathrm{CHF}$ to AHF, for which effective and time-sensitive outcome predictors are urgently needed. ${ }^{14}$ This AHF-specific analysis on the utility of CA125 will complement well with Zhuang et $a l^{15}$ to provide a holistic picture of CA125 as a prognostic marker for CHF as well as AHF.

CA125 is a high-molecular-weight glycoprotein synthesised by coelomic epithelial cells at sites such as the pericardium, pleura and peritoneum. ${ }^{10}$ Although CA125 is classically recognised as a tumour biomarker linked with ovarian cancer, high plasma levels have also been reported in organ failure states, such as HF, nephrotic syndrome, hepatic cirrhosis, previous surgery (including abdominal and non-abdominal surgeries), and pancreatic and gynaecological processes, among others. ${ }^{1137}$ The exact pathogenesis of CA125 elevation in HF is not completely understood. However, preliminary data suggest that activation of mesothelial cells in response to increased hydrostatic pressures, mechanical stress and/or cytokine network activation could act as potential triggers for CA125 secretion. ${ }^{11-13}$

Along this line, plasma levels of CA125 have consistently shown to be related with symptoms or signs of fluid overload, such as peripheral oedemas, serosal effusions, pulmonary wedge pressures and increasing cardiac pressures. ${ }^{11}$ Consistent with this notion, our meta-analysis showed that patients with significant pleural effusions had higher CA125 levels. Furthermore, the present study adds to the literature by showing that CA125 is a useful biomarker for risk stratification purposes, providing incremental value to predict hospitalisations and mortality. Additionally, there are some clinical applications beyond risk stratification that deserve to be commented. First, changes over time of this biomarker have shown to be congruent with clinical status and prognosis, suggesting the utility of glycoprotein for disease monitoring during the first months following a hospitalisation. ${ }^{38}$ Second, a recent clinical trial showed that a CA125-guided therapy in patients with AHF, mostly by modifying diuretic treatment, was superior to conventional therapy in reducing the primary endpoint of composite death and HF readmission at 1-year follow-up. ${ }^{22}$ Based on the previous findings, and given the wide availability, low cost and standardised measurements, we strongly believe that CA125 could play a crucial role during the transition from decompensation to stability in patients with HF. Apart from its demonstrated prognostic potential in HF, the utility of CA125 as a biomarker is predicated on its ready availability, long halflife, reproducibility and broad assessment range. ${ }^{39}$ When it comes to risk stratification and monitoring, CA125 was also considered to be on par with galectin-3 and soluble ST2. ${ }^{11}$ In patients discharged for AHF, it was found that a single determination of BNP at the first ambulatory visit was as effective, in regard to all-cause mortality prediction. However, CA125 was able to provide a temporal perspective allowing for the best discriminative ability compared with either a single or any other change-metrics approach. ${ }^{40}$ Furthermore, the cost of conducting a CA125 assay is significantly (approximately 10 times) cheaper when compared with N-terminal pro b-type natriuretic peptide (NT-proBNP. ${ }^{41}$ 
It is worth noting that literature on differences in the singular prognostic value of CA125 across various aetiologies is limited. However, elevated CA125 has been shown in other cardiac pathologies such as pulmonary hypertension, aortic stenosis and coronary syndromes, ${ }^{42}$ all of which are potential causes of HF. Also, patients used in this meta-analysis had medical histories of cardiac pathologies that could have contributed to $\mathrm{AHF}^{43} 44$

\section{Limitations}

There was a high degree of heterogeneity observed in our meta-analysis. There are several reasons this may be the case. This may be due to different commercially available kits with different reference ranges that were used to determine CA125 levels by the different studies. The majority of these used a solution of suspended, submicron-sized latex particles for its measurements. However, clinical performance for measurement of CA125 elevation has not been compared, and this represents a potential limitation. Moreover, the follow-up durations were not uniform across the studies, ranging from 6 months to 36 months. Furthermore, CA125 varied, largely depending on sex, age, comorbidities including ovarian cancer and chronic obstructive pulmonary disease, and the size of the left atrium (LA). Especially, CA125 level had a strong association with LA size. ${ }^{19} 45$ Also, the different studies included patients with both HF with reduced left ventricular ejection fraction (LVEF) and preserved LVEF. Finally, this study was not a patient-level meta-analysis. Future studies need to carefully account for these potential confounders to better define the relationship between CA125 and acute cardiac decompensation.

\section{CONCLUSIONS}

Our meta-analysis found that high CA125 levels are associated with HF symptoms, HF-related hospital readmissions and all-cause mortality. Therefore, CA125 emerges as a useful tool for risk stratification to identify high-risk patients with more severe fluid overload, as well as for monitoring following an episode of AHF. Based on this meta-analysis, CA125 should be measured for patients with AHF prior to discharge to guide the management plan of the patient. Future cohort studies should look into whether CA125 can be used as an acute prognostic marker within the first few hours within admission.

\footnotetext{
Author affiliations

${ }^{1}$ Department of Medicine and Therapeutics, Faculty of Medicine, Chinese University of Hong Kong, Hong Kong, China

${ }^{2}$ Faculty of Medicine, Li Ka Shing Institute of Health Sciences, Chinese University of Hong Kong, Hong Kong, China

${ }^{3}$ Faculty of Medicine, Newcastle University, England, UK

${ }^{4}$ Department of Cardiology, Tianjin Key Laboratory of Ionic-Molecular Function of Cardiovascular Disease, Tianjin Institute of Cardiology, Second Hospital of Tianjin Medical University, Tianjin, china

${ }^{5}$ Department of Medicine, Kingston General Hospital, Queen's University, Kingston, Ontario, Canada

${ }^{6} \mathrm{JC}$ School of Public Health and Primary Care, The Chinese University of Hong Kong, Hong Kong, China

${ }^{7}$ Heart Institute, Hospital Universitari Germans Trias i Pujol, Badalona, Spain

${ }^{8}$ Department of Medicine, Autonomous University of Barcelona, Barcelona, Spain

${ }^{9}$ Cardiology Department, Hospital Clínico Universitario de Valencia, INCLIVA,

Valencia, Spain

${ }^{10}$ Departamento de Medicina, Universitat de València, Valencia, Spain

${ }^{11}$ CIBER in Cardiovascular Diseases (CIBERCV), Madrid, Spain

${ }^{12}$ Department of Anaesthesia and Intensive Care, State Key Laboratory of Digestive

Disease, The Chinese University of Hong Kong, Hong Kong, China

${ }^{13}$ Shenzhen Research Institute, The Chinese University of Hong Kong, Shenzhen, China
}

\section{Presented at}

The findings of this manuscript were presented at the Inaugural Clinical and Translational Cardiology Conference held in Hong Kong. The abstract will be published by the Journal of Geriatric Cardiology.

Contributors GT: study conception, study screening, data extraction, data analysis, data interpretation, statistical analysis, manuscript drafting, critical revision of the manuscript. MG: study screening, quality analysis, data extraction, manuscript drafting, critical revision of the manuscript. KHCL: study screening, data extraction, data analysis, data interpretation, statistical analysis, critical revision of the manuscript. RWCL, JCLL, GL, AB, TL, MCSW, APWL, ABG, RdIE, JS and AJ: data interpretation, manuscript redrafting, critical revision of the manuscript. WKKW and JN: study conception, study supervision, data interpretation, data interpretation, manuscript redrafting, critical revision of the manuscript.

Funding This work was supported by grants from Centro de Investigación Biomédica en Red de Enfermedades Cardiovasculares (16/11/00420, 16/11/00403), Fondo Europeo de Desarrollo Regional and Proyecto Integrado de Excelencia (PIE15/00013). GT is supported by a Clinical Assistant Professorship from the Croucher Foundation of Hong Kong.

Competing interests JN reports personal fees from Novartis, personal fees from Vifor, personal fees from Abbott, personal fees from Rovi, personal fees from Boehringer Ingelheim and personal fees from Novo Nordisk, outside the submitted work.

\section{Patient consent Not required.}

Provenance and peer review Not commissioned; externally peer reviewed.

\section{REFERENCES}

1 Teerlink JR. Neurohumoral mechanisms in heart failure: a central role for the reninangiotensin system. J Cardiovasc Pharmacol 1996;27(Suppl 2):1-8.

2 Stanley WC, Recchia FA, Lopaschuk GD. Myocardial substrate metabolism in the normal and failing heart. Physiol Rev 2005;85:1093-129.

3 Roger VL. Epidemiology of heart failure. Circ Res 2013;113:646-59.

4 Bundkirchen A, Schwinger RHG. Epidemiology and economic burden of chronic heart failure. Eur Heart J Supp/ 2004;6:D57-D60.

5 Ritzema J, Troughton R, Melton I, et al. Physician-directed patient self-management of left atrial pressure in advanced chronic heart failure. Circulation 2010;121:1086-95.

6. Kommuri NV, Koelling TM, Hummel SL. The impact of prior heart failure hospitalizations on long-term mortality differs by baseline risk of death. Am J Med 2012;125:209.

7 Parrinello G, Torres D, Paterna S, et al. The pathophysiology of acute heart failure: the key role of fluid accumulation. Am Heart J 2008;156:e19.

8 Gheorghiade M, Follath F, Ponikowski P, et al. Assessing and grading congestion in acute heart failure: a scientific statement from the acute heart failure committee of the heart failure association of the European Society of Cardiology and endorsed by the European Society of Intensive Care Medicine. Eur J Heart Fail 2010;12:423-33.

9. Pascual-Figal DA, Caballero L, Sanchez-Mas J, et al. Prognostic markers for acute heart failure. Expert Opin Med Diagn 2013;7:379-92.

10 Topalak 0, Saygili U, Soyturk M. Serum, pleural effusion, and ascites ca-125 levels in ovarian cancer and nonovarian benign and malignant diseases: a comparative study. Gynecol Oncol 2002;85:108-13.

11 Núñez J, Miñana G, Núñez E, et al. Clinical utility of antigen carbohydrate 125 in heart failure. Heart Fail Rev 2014;19:575-84.

12 Huang F, Chen J, Liu Y, et al. New mechanism of elevated CA125 in heart failure: the mechanical stress and inflammatory stimuli initiate CA125 synthesis. Med Hypotheses 2012;79:381-3.

13 Miñana G, Núñez J, Sanchis J, et al. CA125 and immunoinflammatory activity in acute heart failure. Int J Cardiol 2010;145:547-8.

14 Cohen-Solal A, Laribi S, Ishihara S, et al. Prognostic markers of acute decompensated heart failure: the emerging roles of cardiac biomarkers and prognostic scores. Arch Cardiovasc Dis 2015;108:64-74.

15 Zhuang J, Faggiano P, Li Q, et al. Insights into the clinical implications of carbohydrate antigen 125 as a biomarker of heart failure: a meta-analysis and systematic review of published studies. J Cardiovasc Med 2014;15:864-72

16. Moher D, Liberati $A$, et al. PRISMA Group. Preferred reporting items for systematic reviews and meta-analyses: the PRISMA statement. BMJ 2009;339:b2535.

17 Davutoglu V, Yildirim C, Kucukaslan H, et al. Prognostic value of pleural effusion, CA125 and NT-proBNP in patients with acute decompensated heart failure. Kardiol Pol 2010;68:771-8.

18 Durak-Nalbantic A, Resic N, Kulic M, et al. Serum level of tumor marker carbohydrate antigen-CA125 in heart failure. Med Arch 2013;67:241-4.

19 Kaya $H$, Kurt $R$, Beton 0 . Cancer antigen 125 is associated with length of stay in patients with acute heart failure. Tex Heart Inst J 2017;44:22-8. 
20 Kouris NT, Kontogianni DD, Papoulia EP, et al. Clinical and prognostic value of elevated CA125 levels in patients with congestive heart failure. Hellenic J Cardiol 2006;47:269-74.

21 Mansour IN, Napan S, Tarek Alahdab M, et al. Carbohydrate antigen 125 predicts long-term mortality in African American patients with acute decompensated heart failure. Congest Heart Fail 2010;16:15-20.

22 Nunez J, Llacer P, Bertomeu-Gonzalez V. Carbohydrate antigen-125-guided therapy in acute heart failure: Chance-HF: A randomized study. JACC Heart Fail 2016:4:833-43.

23 Núñez J, Rabinovich GA, Sandino J, et al. Prognostic value of the interaction between galectin-3 and antigen carbohydrate 125 in acute heart failure. PLoS One 2015; 10:e0122360.

24 Núñez J, Sanchis J, Bodí V, et al. Improvement in risk stratification with the combination of the tumour marker antigen carbohydrate 125 and brain natriuretic peptide in patients with acute heart failure. Eur Heart J 2010;31:1752-63.

25. Jang SY, Yang DH, Kim CY. Prognostic value of CA-125 in combination with $\mathrm{N}$-terminal pro-brain natriuretic peptide in patients with acute decompensated heart failure. Eur J Heart Fail 2017; 18(Suppl 1):247.

26 Sir JJ, Lee SA, Choi SM. The prognostic value of carbohydrate antigen 125 level in patients with acute decompensated heart failure: Correlation with echocardiographic parameters. Eur J Heart Fail 2017;19:44.

27 Ratkovic N, Danijela V, Slobodan O. Serum concentrations of tumor markers carbohydrate antigen 125 (CA 125) in acute heart failure - clinical implications. Eur J Heart Fail 2016;18:242

28 Santas E, Sandino J, Chorro FJ, et al. Prognostic implications of pericardial effusion in acute heart failure: Does size matter? Int J Cardiol 2015;184:259-61.

29 Nunez J, Nunez E, Minana G. Mortality prediction following an admission for acute heart failure: a longitudinal comparison between $\mathrm{N}$-terminal brain natriuretic peptide and carbohydrate antigen 125. Eur J Heart Fail 2015;17(Suppl 1):261.

30 Josa-Laorden C, Torres-Courchoud I, Martinez-Marin M. Glycoprotein CA125 as short and long term prognostic biomarker in heart failure. Eur J Heart Fail 2015;17(Suppl 1):99.

31 Liu MY, Zhang H, Zhu W. Determining the prognostic value of red cell distribution width and carbohydrate antigen 125 in acute decompensated heart failure patients. Eur J Heart Fail 2013;15(Suppl 1):S130.
32 Bosch Campos MJ, Santas E, Bondanza L. Antigen carbohydrate 125 predicts 30-day readmission in acute heart failure. Eur J Heart Fail 2016;35(Suppl 1):1010.

33 Cheung A, Gong M, Bellanti R, et al. Cancer antigen-125 and risk of atrial fibrillation: a systematic review and meta-analysis. Heart Asia 2018;10:e010970.

34 McQuade CN, Mizus M, Wald JW. Brain-type natriuretic peptide and amino-terminal pro-brain-type natriuretic peptide discharge thresholds for acute decompensated heart failure: A systematic review. Ann Intern Med 2017;166:180-90.

35 Felker GM, Lee KL, Bull DA, et al. Diuretic strategies in patients with acute decompensated heart failure. N Eng/ J Med Overseas Ed 2011;364:797-805.

36 Mecklai A, Subačius H, Konstam MA. In-Hospital diuretic agent use and -discharge clinical outcomes in patients hospitalized for worsening heart failure. JACC Heart Fail 2016:4:580-8.

37 Miralles C, Orea M, España P, et al. Cancer antigen 125 associated with multiple benign and malignant pathologies. Ann Surg Oncol 2003;10:150-4.

38 Núñez J, Nunez E, Bayés-Genís A, et al. Long-term serial kinetics of N-terminal pro B-type natriuretic peptide and carbohydrate antigen 125 for mortality risk prediction following acute heart failure. Eur Heart J Acute Cardiovasc Care 2017;6:685-696.

39 Pacho C, Domingo M, Núñez R, et al. Predictive biomarkers for death and rehospitalization in comorbid frail elderly heart failure patients. BMC Geriatr 2018;18:109.

40 Núñez J, Núñez E, Sanchis J, et al. Antigen carbohydrate 125 and brain natriuretic peptide serial measurements for risk stratification following an episode of acute heart failure. Int J Cardiol 2012;159:21-8.

41 Ordu S, Ozhan H, Alemdar R, et al. Carbohydrate antigen-125 and N-terminal probrain natriuretic peptide levels: compared in heart-failure prognostication. Tex Heart Inst J 2012;39:30-5.

42 Yilmaz MB, Nikolaou M, Cohen Solal A. Tumour biomarkers in heart failure: is there a role for CA-125? Eur J Heart Fail 2011;13:579-83.

43 McMurray JJV, Pfeffer MA. Heart failure. The Lancet 2005;365:1877-89.

44 National Clinical Guideline C. National institute for health and clinical excellence: Guidance. Chronic heart failure: National clinical guideline for diagnosis and management in primary and secondary care: Partial update. 2010.

45 Natanzon A, Kronzon I. Pericardial and pleural effusions in congestive heart failure-anatomical, pathophysiologic, and clinical considerations. Am J Med Sci 2009;338:211-6. 